\title{
The search for alternative splicing regulators: new approaches offer a path to a splicing code
}

\author{
Charles J. David and James L. Manley ${ }^{1}$ \\ Department of Biological Sciences, Columbia University, New York, New York 10027, USA
}

Complex multicellular organisms require a diverse set of proteins to set the form and function of specialized cell types. The availability of complete genomic sequences has revealed that instead of a large increase in the number of protein coding genes compared with unicellular organisms, more complex eukaryotes instead obtain more diversity out of a relatively limited number of genes through the process of alternative splicing (AS). AS results in the cell type-, developmental stage-, sex-, or signal-regulated changes in composition of an mRNA produced from a given gene, brought about by changes in splice site choice (Black 2003; Matlin et al. 2005). There are many different types of AS events, ranging from the tissue-specific inclusion of a cassette exon to the Dscam gene in Drosophila, which contains four clusters of exons containing 12, 48, 33, and 2 mutually exclusive variants, an extreme example of AS complexity (Bharadwaj and Kolodkin 2006). Additionally, it is clear from the relatively small number of AS events that have been studied extensively at a mechanistic level that regulation of AS takes many forms, as will be discussed in more detail later in this article. Lastly, the functional outcomes of AS vary greatly, from effectively turning off a gene (the result of including an exon containing a premature stop codon, for example), to a subtle change in a protein's function.

In spite of this complexity, the goal of understanding the changes in AS patterns in terms of changes in expression and regulation of factors that regulate AS across cell types appears achievable. This is in part because recent technical advances allow us, starting with an individual splicing factor, to determine its genome-wide role in AS regulation. However, the ambitious goal of determining a cellular code for AS will be impossible to realize without a complete list of AS regulators, and currently there is no reason to believe we are anywhere near completing such a list in any metazoan.

Kuroyanagi et al. (2006) have developed recently a system in Caenorhabditis elegans, which allows for the

${ }^{1}$ Corresponding author.

E-MAIL jlm2@columbia.edu; FAX (212) 865-8246.

Article is online at http://www.genesdev.org/cgi/doi/10.1101/gad.1643108. straightforward visualization of individual AS events in vivo, which in turn allows for the identification of mutants defective in regulating the event. Using this approach, Kuroyanagi and colleagues (Kuroyanagi et al. 2006, 2007; Ohno et al. 2008) have identified four proteins that regulate two different AS events, a feat that would be laborious using in vitro approaches. Three of these proteins had not been shown previously to participate in regulation of AS, indicating that we may be far from a complete list of AS regulators. Their data also highlight the fact that regulators of AS are often highly conserved throughout metazoans, meaning that filling the list of splicing regulators in C. elegans will likely contribute to filling our own.

\section{Known AS regulators}

There are a large number of factors known to be involved in AS, which can be crudely lumped into two classes. One class of AS regulators consists of relatively widely expressed proteins, which seem to have wide-ranging roles in mRNA biogenesis. These come in two groups: SR proteins, which, when bound to exons, tend to promote exon inclusion, and hnRNP proteins, which usually have the opposite effect (Manley and Tacke 1996; Graveley 2000; Smith and Valcarcel 2000). While these proteins are widely expressed and have roles in numerous processes, cell-type-specific changes in their expression levels or post-translational modifications that alter their activities or localization provide a means of AS regulation. For example, hnRNP A1, which inhibits the inclusion of many alternative exons, becomes phosphorylated upon osmotic shock resulting in its cytoplasmic accumulation, which in turn affects a number of AS events (Allemand et al. 2005). While we are very far from knowing all the activities of the hnRNP and SR proteins in AS regulation, it is safe to assume that all members of these widely expressed families have been identified, although some tissue-specific isoforms relevant to AS may yet be discovered.

No such confident statements can be made about the other broad class of AS regulators, proteins with a much narrower job description. These are factors with re- 
stricted expression patterns that are responsible for regulating tissue-specific AS events. The idea that factors of restricted expression regulate specific AS events has been around since the elucidation of the Drosophila sex determination pathway. This pathway begins with a female-specific RNA-binding protein, Sxl, which regulates the splice site choices of a small number of transcripts that are alternatively spliced in a sex-specific manner (Lopez 1998). Additional tissue-specific factors (often small families of factors) that play a role in regulating AS of a relatively small number of transcripts have been identified and characterized to varying extents. In mammals, these include Nova-1/2 (Jensen et al. 2000), TIA1/ TIAR (Del Gatto-Konczak et al. 2000; Forch et al. 2000), Fox-1/2 (Jin et al. 2003), nPTB (Markovtsov et al. 2000), STAR/GSG family proteins (Butcher and Wickens 2004), Tra $2 \alpha / \beta$ (Tacke et al. 1998), CELF family proteins (Ladd et al. 2001), and Hu proteins (Zhu et al. 2006). These proteins have been identified by a wide variety of approaches, and each of the factors listed shares a salient feature: Each contains an RNA-binding domain of the KH or RRM type.

For many of the specific AS regulators we currently know, the initial implication in AS was based solely on the presence of an RNA-binding motif. Using databases made possible by complete genomic sequence available in mouse, McKee et al. (2005) sought to compile a comprehensive list of mouse RNA-binding proteins based on known RNA-binding motifs. The resulting set contained 380 proteins, most of which contained RRMs or KH domains (McKee et al. 2005). While this is a large number, it does not approach that of transcription factors, which, according to some estimates, number up to 2500 (Tupler et al. 2001). McKee et al. (2005) showed that 221 of these putative RNA-binding proteins exhibit regionally restricted expression in the brain. A large number of the proteins identified as putative RNA-binding proteins were minimally characterized, or not characterized at all. So while the list of known specific AS regulators in mammals numbers under two dozen, this genome-wide analysis suggests that there is room for that number to expand. It should be pointed out that AS regulators do not need to contain an RNA-binding domain to control specific AS events; here the Drosophila sex determination pathway offers another lesson. The female-specific Tra protein, devoid of any known RNA-binding domain, is required for the cooperative assembly of RBP1 and other SR proteins, as well as the SR-related protein Tra2, on the dsx enhancer element (Tian and Maniatis 1994). This type of regulation, analogous to that of transcriptional coactivators, has also been demonstrated in humans. SRm160, an RS domain-containing splicing factor without an apparent RNA-binding domain, participates in the regulation of $C D 44$ variable exon 5 splicing (Cheng and Sharp 2006).

Also of interest is the potential influence of transcription itself on AS, and the possibility that transcription factors might serve as AS regulators. A decade ago, Kornblihtt and colleagues (Cramer et al. 1997) made the observation that the identity of the promoter driving a given transiently transfected minigene encoding an alternatively spliced transcript can alter the outcome of the AS event. This finding raised the intriguing possibility that transcription factors could serve as AS regulators, either by influencing the concentration of direct regulators of AS within the alternatively spliced gene's transcriptional milieu, or by altering the rate of RNA polymerase II elongation, leading to indirect effects on AS (Kornblihtt 2005). An example of a transcription factor that can influence splicing was provided by the mammalian protein PGC-1, a transcriptional coactivator involved in adaptive thermogenesis. This protein, in addition to domains relevant to transcription, contains an RRM as well as an RS-rich domain, both characteristic of splicing factors, and these apparently influence the splicing of transcripts it regulates (Monsalve et al. 2000). Identifying instances in which tissue-specific changes in transcription factor expression alter AS patterns in a direct and physiologically relevant way will be of interest in the future.

Most of the factors listed above as tissue-specific AS regulators were of interest prior to their implication in AS. In fact, very few AS factors have been first characterized solely on the basis of their participation in AS. Identifying regulators of a particular AS event, starting from scratch, is difficult. A blueprint for this approach is provided by the work of Black and colleagues on the neuron-specific inclusion of the c-src N1 exon. The first step was the reconstitution of the AS pattern in cultured cells (Black 1992). Next, cis-elements required for the regulation of the event were mapped (Black 1992; Chan and Black 1995; Modafferi and Black 1997). These sequences in hand, RNA affinity chromatography was used to identify factors that bind to these sequences in nuclear extracts, which led to the identification of two novel AS regulators, KSRP and nPTB (Min et al. 1997; Markovtsov et al. 2000). While this approach was successful in identifying novel AS regulators, it is too involved to be applied to a large number of AS events. Another way of identifying regulators of particular AS events is through RNAi screens (Celotto and Graveley 2004). Genome-wide screens already have been applied to other mRNA processing events (Wagner et al. 2007), and this approach will certainly prove useful in the analysis of AS regulation. One shortcoming of this approach is that AS events must first be recapitulated in cell culture, which may not always be possible.

\section{A genetic approach to identifying AS regulators in C. elegans}

Kuroyanagi et al. (2006) have developed a system that allows for the visualization of AS decisions in intact organisms. The method involves the use of minigene reporters that express red fluorescent protein (RFP) or green fluorescent protein (GFP), dependent on the cellspecific outcome of the AS event being studied. In this issue of Genes and Development, Ohno et al. (2008) apply this method to the mutually exclusive splicing of 
let-2 exons 9/10. Let-2 encodes $\alpha 2(\mathrm{IV})$ collagen in $C$. elegans, and expression undergoes a switch during development, from an mRNA isoform that contains exon 9 (E9) in embryos to an isoform that instead contains exon 10 (E10) during late larval stages and in the adult worm. This AS event was first reported 15 years ago, but until now nothing was known about the factors that regulate it (Sibley et al. 1993).

Ohno et al. (2008) constructed two reporter minigenes, each containing either GFP or RFP downstream from a region of the let-2 gene spanning exon 8 to exon 11, and under the control of the body wall-specific promoter myo3. To construct a minigene that produces GFP when E9 is selected, Ohno et al. (2008) altered a single nucleotide in E10 to introduce a stop codon upstream of GFP, should it be included. In the other minigene, the stop codon was instead introduced in E9, upstream of RFP, resulting in RFP expression only when E10 is selected. Worms containing these two minigenes showed a distinct switch from green in embryos to red in more mature organisms, in a way that precisely matched the switch from E9 to E10 usage in the endogenous let-2 transcript.

Armed with the ability to monitor visually the E9/E10 switching phenotype, Ohno et al. (2008) next screened for mutants defective in the regulation of this event. Of the several independent mutant alleles they identified, all resulted in worms that remained green throughout development. Remarkably, all of the mutations were in the same uncharacterized gene, which Ohno et al. (2008) named alternative-splicing-defective-2 (asd-2). Expressed sequence tag (EST) analysis indicated that this locus produced two mRNA isoforms containing alternate first exons, predicted to result in proteins differing at the $\mathrm{N}$ terminus, ASD-2a and ASD-2b. Of these, ASD$2 \mathrm{~b}$ was the only isoform detected in body wall muscles. Three null alleles identified were predicted to specifically disrupt the $2 \mathrm{~b}$ isoform, while another five were missense mutations that fell within a conserved GSG/ STAR domain (see below). Overexpression of ASD-2b in body wall muscles led to increased E10 inclusion earlier in development than normally observed, while RNAi depletion of ASD-2b phenocopied the original asd-2 mutants.
The GSG/STAR domain is the signature of the STAR family of RNA-binding proteins, a highly conserved group of multifunctional regulators of gene expression found throughout metazoans. These include the C. elegans protein GLD-1, known to mediate translational repression of some target mRNAs (Schumacher et al. 2005), and quaking (QKI), which has been shown to regulate AS in mammals (Wu et al. 2002; Schumacher et al. 2005). A QKI high-affinity binding site has been determined in vitro and named the quaking response element (QRE) (Galarneau and Richard 2005). Galarneau and Richard also observed that previously identified GLD-1-binding sites fall within QREs, demonstrating conservation of RNA-binding specificities among the STAR family.

Previous studies have indicated that a short stretch of let-2 intron 10, conserved between C. elegans and Caenorhabditis briggsae, is required for the proper regulation of exon 9/10 splicing (Kabat et al. 2006). Inspection of this region revealed a consensus QRE, to which ASD-2b indeed bound in vitro. Disruption of the consensus QRE by two point mutations drastically reduced ASD-2b binding in vitro, phenocopied the splicing defect of the asd-2 mutants isolated in the original screen, and failed to respond to ASD-2b overexpression, making a persuasive case that ASD-2b functions through this element to promote the switch from E9 to E10 inclusion during development. An additional observation, however, makes it clear that we are not yet privy to the whole story: ASD-2b is uniformly expressed throughout development. One potential explanation for the inactivity of ASD-2b early in development is that it is subject to post-translational modification that regulates its RNA-binding activity, as is the case for the closely related mammalian protein Sam68 (Matter et al. 2002). Another possibility is that ASD-2b requires another factor to stabilize its RNA binding, while a third possibility is that ASD-2b, once bound, cooperates with another factor to promote exon 10 inclusion (discussed below).

Analysis of the splicing intermediates in the region spanning exons 8-11 provides some insight into the mechanism by which ASD-2b functions in promoting the switch from E9 to E10. In wild-type embryos, a species retaining intron 8 in which E9 and E11 are ligated

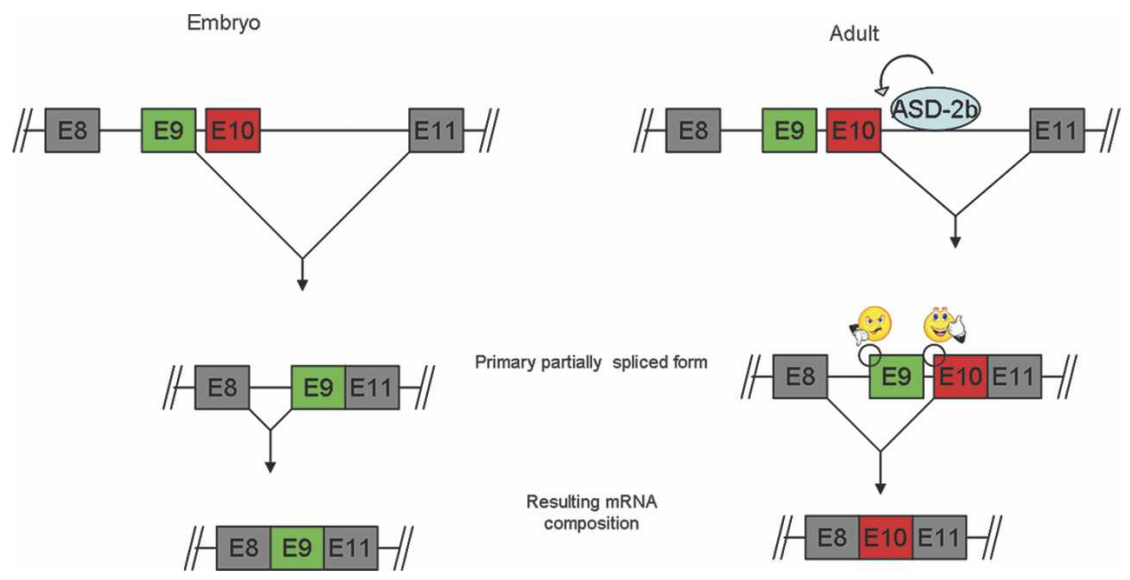

Figure 1. ASD-2b promotes E10 inclusion by altering $5^{\prime}$ splice site usage in the intron downstream from the regulated exons. In embryos, E10 is removed in a fast splicing event, leaving a product with E9 and E11 ligated together as the predominant partially spliced isoform, which is processed to remove intron 8 in a slower reaction. In adults, ASD- $2 b$, through an unknown mechanism, promotes usage of the E10 donor site in the fast reaction. This results in a competition between E9 and E10 acceptor sites. E9 is removed because it lies downstream from a weaker $3^{\prime}$ splice site. 
together accounts for nearly all partially spliced RNA. In L4 stage worms, this species is greatly reduced, and instead a form in which E10 and E11 are joined predominates (Fig. 1). In this intermediate, the E9 and E10 splice acceptors are in competition. The E10 acceptor site is favored in this competition, apparently because the region upstream of it contains a better binding site for the general splicing factor U2AF65 than the region upstream of E9. The E10/E11 intermediate is therefore exclusively processed to remove E9. In asd-2 mutants, however, the predominant partially spliced form throughout development remains the E9/E11 form. This suggests that the primary function of ASD-2b in effecting the switch from E9 to E10 is mediated by binding to an intronic site and promoting the joining of E10 with E11. This is not the end of the story, as Ohno et al. (2008) also note that there is an increase in the amount of an E8/E10 splicing intermediate in adult worms that is not significantly decreased in the asd-2 mutants, indicating that additional factors are at play in promoting the E9-to-E10 switch. The additional factor(s) regulating this event may be essential for early embryonic development in C. elegans, which would explain its failure to be detected in the mutant screen.

What is the mechanism by which ASD-2b mediates exon inclusion? The activity of ASD-2b in let-2 splicing is reminiscent of a number of other AS factors that bind downstream from alternative exons they positively regulate. One regulator, TIA1, functions by directly interacting with U1 snRNP to recruit it to the upstream 5 ' splice site (Fig. 2; Del Gatto-Konczak et al. 2000; Forch et al. 2000). This mechanism was originally predicted because TIA1 is homologous to the yeast U1 snRNP protein Nam8p (Del Gatto-Konczak et al. 2000; Forch et al. 2000). It may be that ASD-2b functions in a related way. However, given the diversity of other AS regulators that also function by binding to downstream introns, it is possible that not all function through direct interactions with the splicing machinery. Instead, some intron-bound AS regulators may act by intron definition. This could occur through an interaction between proteins bound at either end of the intron downstream from the regulated exon, leading to the looping out of intervening intronic sequence, bringing the regulated exon's donor site into proximity with the downstream acceptor site (Fig. 2). This mechanism has been suggested for some instances of splicing enhancement mediated by hnRNPs binding to intronic splicing enhancers, as well as for the Nova proteins (Martinez-Contreras et al. 2006; Ule et al. 2006). If this is the case in let-2 regulation, other conserved regions of intron 10 that do not bind ASD-2b may come into play, along with (an) additional factor(s) not identified in the screen, offering another conceivable explanation for why ASD-2b expression throughout development is insufficient to affect splicing early in development.

\section{Three conserved splicing regulators identified in another AS event}

Kuroyanagi et al. $(2006,2007)$ recently used the same approach to study another AS event: tissue-specific expression egl-15 E5A-containing isoform, rather than the alternative E5B isoform, in sex myoblasts, daughter cells of which are destined to become body wall muscles, as well as specialized sex-specific muscles later in development. Mutant screening revealed three regulators of this event: FOX-1; ASD-1, a previously uncharacterized member of the Fox-1 family; and SUP-12, an RRM-containing protein previously shown to regulate AS (Anyanful et al. 2004; Kuroyanagi et al. 2006, 2007). In another example of the conservation of AS regulators and the sequences to which they bind, FOX-1 and ASD-1 were shown to function through a conserved UGCAUG se-
Figure 2. Potential mechanisms for exon inclusion stimulated by AS regulators binding to the downstream intron. In the recruitment pathway, the intron-bound AS regulator promotes exon recognition through specific contacts with the splicing machinery. TIA1 recruitment of U1 snRNP to $5^{\prime}$ splice sites of exons that it regulates serves as an example. In the conformational pathway, regulators bound to an intronic site promote regulated exon inclusion by intron definition. This might involve an interaction between regulators bound at either end of the intron that would serve to loop out intronic sequences and bring the regulated exon into proximity with the downstream constitutive exon, thereby favoring its inclusion.

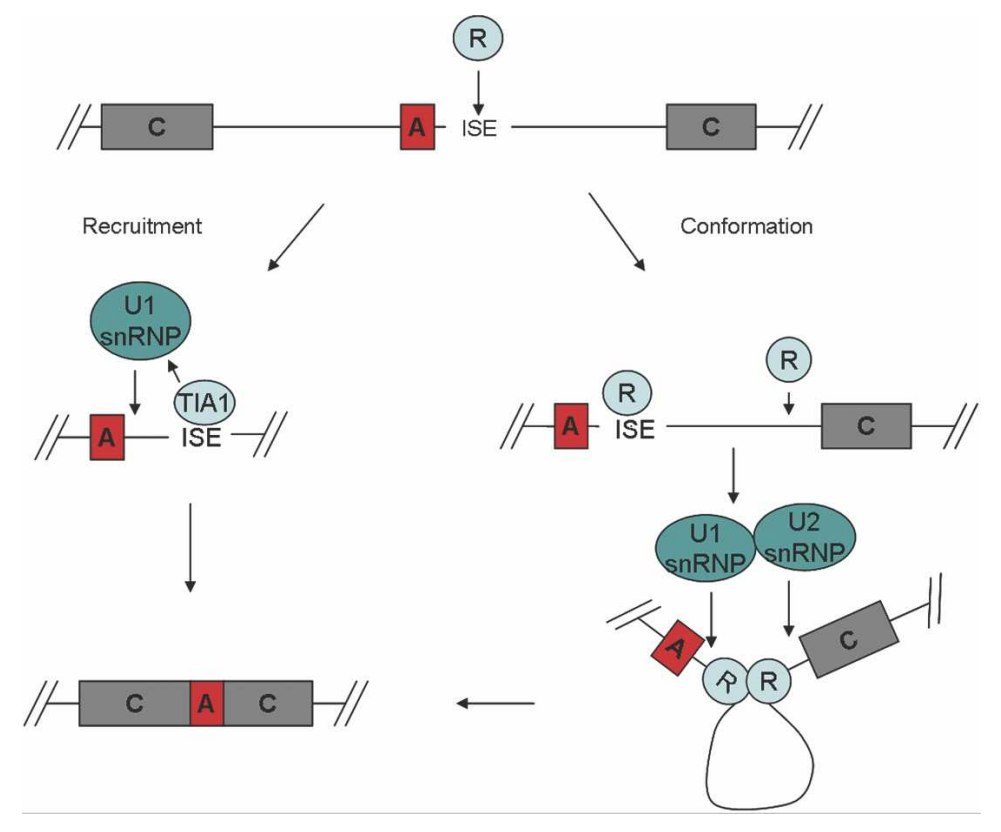


quence located upstream of the mutually exclusive exons. This sequence is identical to that bound by mammalian Fox-1/2 (Jin et al. 2003). This is unsurprising, since all residues shown to mediate the specific recognition of the UGCAUG sequence in human Fox-1 are conserved in the C. elegans proteins (Jin et al. 2003; Auweter et al. 2006). FOX-1 and ASD-1 are more widely expressed than the E5A isoform, and therefore their expression pattern does not explain the tissue specificity of the egl-15 AS. Additional screening revealed another factor required for the expression of the $\mathrm{E} 5 \mathrm{~A}$ isoform, SUP-12. SUP-12 is a muscle-specific protein that cooperates with FOX-1 and ASD-1 to bind stably to the egl-15 pre-mRNA and promote the inclusion of E5A (Kuroyanagi et al. 2007). SUP-12 and its poorly characterized human homolog RNPC1 share a higher degree of identity than even the Fox-1 family. Given the high degree of conservation between other known AS regulators, RNPC1 is highly likely to be an AS regulator in mammals.

\section{From the identification of AS regulators to a cellular code of AS regulation}

Thanks to some important technical developments, known AS regulators, as well as potential ones such as RNPC1, can be evaluated for their transcriptome-wide RNA binding. From this, their contribution to the AS of alternative exons can be predicted genome-wide. This is due in part to the development of the cross-linking and immunoprecipitation (CLIP), a procedure pioneered by Darnell and colleagues (Ule et al. 2003). Briefly, CLIP involves the UV irradiation of a tissue of interest to induce cross-links between RNA and bound proteins. Following light RNase treatment to reduce the average length of RNA fragments to $\sim 50$ nucleotides, the protein of interest is immunoprecipitated under stringent conditions and then digested with proteinase $\mathrm{K}$, leaving RNA that can be amplified and cloned (Ule et al. 2003), hybridized to a microarray, or amplified and sequenced directly, now using high-throughput technology (J. Sanford, pers. comm.). The latter two approaches have the potential to identify the genome-wide distribution of a given factor. As has been demonstrated with Nova, an RNA map of this kind makes it possible to predict the effect that a given AS regulator has in regulating individual AS events based on binding and splicing patterns observed in a much smaller subset of genes (Ule et al. 2006). In the case of Nova, binding sites within alternate exons predicted its negative regulation of that exon, whereas binding at specific sites within the flanking introns was predictive of positive regulation, raising the possibility that AS regulators function through nonspecific mechanisms based solely on the position of their binding sites. It will be of great interest to create similar RNA-binding maps for additional factors that regulate AS, both the ubiquitous (SR proteins and hnRNPs) and the tissue-specific proteins. In light of the diversity of AS regulators that function through intronic binding sites to positively regulate exon inclusion, the identification of shared patterns between different families of factors may illuminate the mechanisms through which they work. Additional methods such as RNP immunoprecipitationmicroarray (RIP-Chip) will supplement CLIP to provide complete maps of RNA-binding factor distribution (Keene et al. 2006).

\section{Conclusions and perspectives}

Methods such as CLIP have made it possible to map RNA-binding factors throughout the genome, while the advent of technologies such as AS microarrays have also greatly increased the number of AS events of which we are aware (e.g., see Pan et al. 2004). The combination of the two approaches may prove useful in explaining the tissue specificity of a large number of AS events, but if our catalog of regulators is not complete, it will not explain all AS events.

In contrast to previous approaches, the major advantage to the system developed by Kuroyanagi et al. (2006) is that it can be widely applied to additional AS events in C. elegans, as well as other genetically tractable models such as Drosophila. Because so many of the AS regulators identified using this method in C. elegans have clear mammalian homologs, this approach will certainly prove useful in identifying candidate regulators in humans. Widespread use of this system is likely to unearth many additional regulators of AS that are currently on the long list of uncharacterized proteins containing an RNA-binding domain. We may also be reminded that, as we learned long ago in a genetic screen for Drosophila sex determination genes, an RNA-binding domain is not a requirement for membership in the club of AS regulators. While the complexity of AS is at first glance daunting, systematic approaches to identifying regulators of AS combined with novel methods that allow us to assess the genome-wide activity of individual splicing factors, mean that elucidation of an AS code may be feasible.

\section{Acknowledgments}

We thank members of the Manley laboratory for helpful discussion.

\section{References}

Allemand, E., Guil, S., Myers, M., Moscat, J., Caceres, J.F., and Krainer, A.R. 2005. Regulation of heterogenous nuclear ribonucleoprotein A1 transport by phosphorylation in cells stressed by osmotic shock. Proc. Natl. Acad. Sci. 102: 36053610.

Anyanful, A., Ono, K., Johnsen, R.C., Ly, H., Jensen, V., Baillie, D.L., and Ono, S. 2004. The RNA-binding protein SUP-12 controls muscle-specific splicing of the ADF/cofilin premRNA in C. elegans. J. Cell Biol. 167: 639-647.

Auweter, S.D., Fasan, R., Reymond, L., Underwood, J.G., Black, D.L., Pitsch, S., and Allain, F.H. 2006. Molecular basis of RNA recognition by the human alternative splicing factor Fox-1. EMBO J. 25: 163-173.

Bharadwaj, R. and Kolodkin, A.L. 2006. Descrambling Dscam diversity. Cell 125: 421-424.

Black, D.L. 1992. Activation of c-src neuron-specific splicing by 
an unusual RNA element in vivo and in vitro. Cell 69: 795807.

Black, D.L. 2003. Mechanisms of alternative pre-messenger RNA splicing. Annu. Rev. Biochem. 72: 291-336.

Butcher, S.E. and Wickens, M. 2004. STAR-studded circuitry. Nat. Struct. Mol. Biol. 11: 2-3.

Celotto, A.M. and Graveley, B.R. 2004. RNA interference of mRNA processing factors in Drosophila S2 cells. Methods Mol. Biol. 257: 245-254.

Chan, R.C. and Black, D.L. 1995. Conserved intron elements repress splicing of a neuron-specific c-src exon in vitro. Mol. Cell. Biol. 15: 6377-6385.

Cheng, C. and Sharp, P.A. 2006. Regulation of CD44 alternative splicing by SRm160 and its potential role in tumor cell invasion. Mol. Cell. Biol. 26: 362-370.

Cramer, P., Pesce, C.G., Baralle, F.E., and Kornblihtt, A.R. 1997. Functional association between promoter structure and transcript alternative splicing. Proc. Natl. Acad. Sci. 94: 11456-11460.

Del Gatto-Konczak, F., Bourgeois, C.F., Le Guiner, C., Kister, L., Gesnel, M.C., Stevenin, J., and Breathnach, R. 2000. The RNA-binding protein TIA-1 is a novel mammalian splicing regulator acting through intron sequences adjacent to a $5^{\prime}$ splice site. Mol. Cell. Biol. 20: 6287-6299.

Forch, P., Puig, O., Kedersha, N., Martinez, C., Granneman, S., Seraphin, B., Anderson, P., and Valcarcel, J. 2000. The apoptosis-promoting factor TIA-1 is a regulator of alternative pre-mRNA splicing. Mol. Cell 6: 1089-1098.

Galarneau, A. and Richard, S. 2005. Target RNA motif and target mRNAs of the Quaking STAR protein. Nat. Struct. Mol. Biol. 12: 691-698.

Graveley, B.R. 2000. Sorting out the complexity of SR protein functions. RNA 6: 1197-1211.

Jensen, K.B., Dredge, B.K., Stefani, G., Zhong, R., Buckanovich, R.J., Okano, H.J., Yang, Y.Y., and Darnell, R.B. 2000. Nova-1 regulates neuron-specific alternative splicing and is essential for neuronal viability. Neuron 25: 359-371.

Jin, Y., Suzuki, H., Maegawa, S., Endo, H., Sugano, S., Hashimoto, K., Yasuda, K., and Inoue, K. 2003. A vertebrate RNAbinding protein Fox-1 regulates tissue-specific splicing via the pentanucleotide GCAUG. EMBO J. 22: 905-912.

Kabat, J.L., Barberan-Soler, S., McKenna, P., Clawson, H., Farrer, T., and Zahler, A.M. 2006. Intronic alternative splicing regulators identified by comparative genomics in nematodes. PLoS Comput. Biol. 2: e86. doi: 10.1371/journal.pcbi. 0020086.

Keene, J.D., Komisarow, J.M., and Friedersdorf, M.B. 2006. RIP-Chip: The isolation and identification of mRNAs, microRNAs and protein components of ribonucleoprotein complexes from cell extracts. Nat. Protoc. 1: 302-307.

Kornblihtt, A.R. 2005. Promoter usage and alternative splicing. Curr. Opin. Cell Biol. 17: 262-268.

Kuroyanagi, H., Kobayashi, T., Mitani, S., and Hagiwara, M. 2006. Transgenic alternative-splicing reporters reveal tissuespecific expression profiles and regulation mechanisms in vivo. Nat. Methods 3: 909-915.

Kuroyanagi, H., Ohno, G., Mitani, S., and Hagiwara, M. 2007. The Fox-1 family and SUP-12 coordinately regulate tissuespecific alternative splicing in vivo. Mol. Cell. Biol. 27: 8612-8621.

Ladd, A.N., Charlet, N., and Cooper, T.A. 2001. The CELF family of RNA binding proteins is implicated in cell-specific and developmentally regulated alternative splicing. Mol. Cell. Biol. 21: 1285-1296.

Lopez, A.J. 1998. Alternative splicing of pre-mRNA: Developmental consequences and mechanisms of regulation. Annu.
Rev. Genet. 32: 279-305.

Manley, J.L. and Tacke, R. 1996. SR proteins and splicing control. Genes \& Dev. 10: 1569-1579.

Markovtsov, V., Nikolic, J.M., Goldman, J.A., Turck, C.W., Chou, M.Y., and Black, D.L. 2000. Cooperative assembly of an hnRNP complex induced by a tissue-specific homolog of polypyrimidine tract binding protein. Mol. Cell. Biol. 20: 7463-7479.

Martinez-Contreras, R., Fisette, J.F., Nasim, F.U., Madden, R., Cordeau, M., and Chabot, B. 2006. Intronic binding sites for hnRNP A/B and hnRNP F/H proteins stimulate pre-mRNA splicing. PLOS Biol. 4: e21. doi: 10.1371/journal.pbio. 0040021 .

Matlin, A.J., Clark, F., and Smith, C.W. 2005. Understanding alternative splicing: Towards a cellular code. Nat. Rev. Mol. Cell Biol. 6: 386-398.

Matter, N., Herrlich, P., and Konig, H. 2002. Signal-dependent regulation of splicing via phosphorylation of Sam68. Nature 420: 691-695.

McKee, A.E., Minet, E., Stern, C., Riahi, S., Stiles, C.D., and Silver, P.A. 2005. A genome-wide in situ hybridization map of RNA-binding proteins reveals anatomically restricted expression in the developing mouse brain. BMC Dev. Biol. 5: 14. doi: 10.1186/1471-213x-5-14.

Min, H., Turck, C.W., Nikolic, J.M., and Black, D.L. 1997. A new regulatory protein, KSRP, mediates exon inclusion through an intronic splicing enhancer. Genes \& Dev. 11: 1023-1036.

Modafferi, E.F. and Black, D.L. 1997. A complex intronic splicing enhancer from the c-src pre-mRNA activates inclusion of a heterologous exon. Mol. Cell. Biol. 17: 6537-6545.

Monsalve, M., Wu, Z., Adelmant, G., Puigserver, P., Fan, M., and Spiegelman, B.M. 2000. Direct coupling of transcription and mRNA processing through the thermogenic coactivator PGC-1. Mol. Cell 6: 307-316.

Ohno, G., Hagiwara, M., and Kuroyanagi, H. 2008. STAR family RNA-binding protein ASD-2 regulates developmental switching of mutually exclusive alternative splicing in vivo. Genes \& Dev. (this issue), doi: 10.1101/gad.1620608.

Pan, Q., Shai, O., Misquitta, C., Zhang, W., Saltzman, A.L., Mohammad, N., Babak, T., Siu, H., Hughes, T.R., Morris, Q.D., et al. 2004. Revealing global regulatory features of mammalian alternative splicing using a quantitative microarray platform. Mol. Cell 16: 929-941.

Schumacher, B., Hanazawa, M., Lee, M.H., Nayak, S., Volkmann, K., Hofmann, E.R., Hengartner, M., Schedl, T., and Gartner, A. 2005. Translational repression of C. elegans p53 by GLD-1 regulates DNA damage-induced apoptosis. Cell 120: $357-368$.

Sibley, M.H., Johnson, J.J., Mello, C.C., and Kramer, J.M. 1993. Genetic identification, sequence, and alternative splicing of the Caenorhabditis elegans $\alpha$ 2(IV) collagen gene. J. Cell Biol. 123: 255-264.

Smith, C.W. and Valcarcel, J. 2000. Alternative pre-mRNA splicing: The logic of combinatorial control. Trends Biochem. Sci. 25: 381-388.

Tacke, R., Tohyama, M., Ogawa, S., and Manley, J.L. 1998. Human Tra2 proteins are sequence-specific activators of premRNA splicing. Cell 93: 139-148.

Tian, M. and Maniatis, T. 1994. A splicing enhancer exhibits both constitutive and regulated activities. Genes \& Dev. 8: 1703-1712.

Tupler, R., Perini, G., and Green, M.R. 2001. Expressing the human genome. Nature 409: 832-833.

Ule, J., Jensen, K.B., Ruggiu, M., Mele, A., Ule, A., and Darnell, R.B. 2003. CLIP identifies Nova-regulated RNA networks in 
the brain. Science 302: 1212-1215.

Ule, J., Stefani, G., Mele, A., Ruggiu, M., Wang, X., Taneri, B., Gaasterland, T., Blencowe, B.J., and Darnell, R.B. 2006. An RNA map predicting Nova-dependent splicing regulation. Nature 444: 580-586.

Wagner, E.J., Burch, B.D., Godfrey, A.C., Salzler, H.R., Duronio, R.J., and Marzluff, W.F. 2007. A genome-wide RNA interference screen reveals that variant histones are necessary for replication-dependent histone pre-mRNA processing. Mol. Cell 28: 692-699.

Wu, J.I., Reed, R.B., Grabowski, P.J., and Artzt, K. 2002. Function of quaking in myelination: Regulation of alternative splicing. Proc. Natl. Acad. Sci. 99: 4233-4238.

Zhu, H., Hasman, R.A., Barron, V.A., Luo, G., and Lou, H. 2006. A nuclear function of $\mathrm{Hu}$ proteins as neuron-specific alternative RNA processing regulators. Mol. Biol. Cell 17: 51055114. 


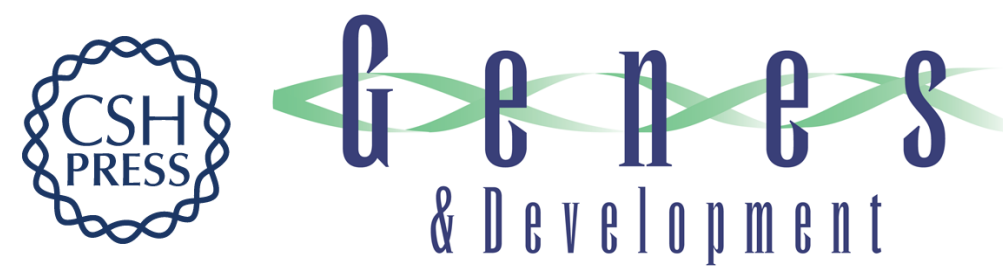

\section{The search for alternative splicing regulators: new approaches offer a path to a splicing code}

Charles J. David and James L. Manley

Genes Dev. 2008, 22:

Access the most recent version at doi:10.1101/gad.1643108

\section{Related Content STAR family RNA-binding protein ASD-2 regulates developmental switching of mutually exclusive alternative splicing in vivo \\ Genta Ohno, Masatoshi Hagiwara and Hidehito Kuroyanagi \\ Genes Dev. February , 2008 22: 360-374}

References This article cites 46 articles, 20 of which can be accessed free at:

http://genesdev.cshlp.org/content/22/3/279.full.html\#ref-list-1

Articles cited in:

http://genesdev.cshlp.org/content/22/3/279.full.html\#related-urls

\section{License}

Email Alerting

Service

Receive free email alerts when new articles cite this article - sign up in the box at the top right corner of the article or click here.

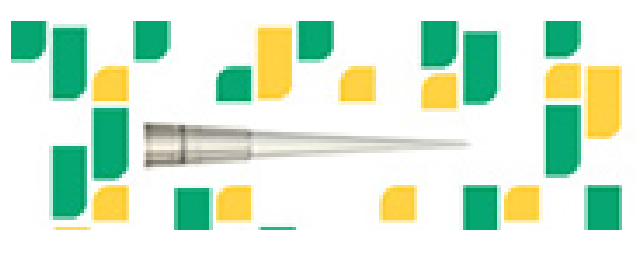

Focused on your science. 